\title{
LA MACRO-COMPARACIÓN ENTRE LA CICIG Y LA MACCIH, UNA NECESIDAD COMPARTIDA Y DIFERENCIADA
}

\section{Juan Carlos Murillo Castellanos ${ }^{1}$}

DOI: https://doi.org/10.5377//rd.v41i1.10487

\section{RESUMEN}

El estudio se centra en comparar desde una perspectiva jurídico-científica el rol de la Comisión Internacional Contra la Impunidad en Guatemala (CICIG) y de la Misión de Apoyo Contra la Corrupción y la Impunidad en Honduras (MACCIH). En esta macro-comparación se develan tanto sus aspectos coincidentes, como divergentes, y la complejidad contextual dentro de cada realidad social. Asimismo, el marco analítico que ha permitido calificar su incidencia de manera efectiva, y una reflexión objetiva sobre sus implicaciones para revelar una mirada alternativa al Status Quo que impera en ambas sociedades.

\section{PALABRAS CLAVE:}

Macro-comparación, CICIG, MACCIH, Corrupción, Impunidad

Fecha de recepción: 08 de agosto del 2020 Fecha de aprobación:22 de octubre de 2020

1 Doctor en Desarrollo Local y Cooperación Internacional por la Universitat Politécnica de Valencia (UPV) y Máster en Derecho Corporativo o Empresarial por la Universidad Tecnológica Centroamericana (UNITEC), correo electrónico juanmurillo3000@ gmail.com. 


\title{
THE MACRO-COMPARISON BETWEEN THE CICIG AND THE MACCIH, A SHARED AND DIFFERENTIATED NECESSITY
}

\author{
Juan Carlos Murillo Castellanos ${ }^{2}$ \\ DOI: $\underline{\text { https://doi.org/10.5377//rd.v41i1.10487 }}$
}

\section{ABSTRACT}

This study focuses on comparing from a legal-scientific perspective the role of the International Commission Against Impunity in Guatemala (CICIG) and the Mission of Support Against Corruption and Impunity in Honduras (MACCIH). In this macro-comparison, both its coincident and divergent aspects are revealed, as well as the contextual complexity within each social reality. Likewise, the analytical framework that has allowed us to qualify its incidence effectively, and an objective reflection on its implications to reveal an alternative view of the Status Quo that prevails in both societies.

\section{KEY WORDS:}

Macro-comparison, CICIG, MACCIH, Corruption, Impunity

Date received: August 08, 2020

Approval date: October 22, 2020

\footnotetext{
2 Doctor in Local Development and International Cooperation from the Polytechnic University of Valencia (UPV) and Master's Degree in Corporate or Business Law from the Central American Technological University (UNITEC),

Emai: juanmurillo3000@gmail.com.
} 


\section{INTRODUCCIÓN}

En la actualidad la corrupción y la impunidad se han acentuado en naciones caracterizadas por la fragilidad de sus sistemas democráticos (Aidt, 2010; World Bank, 2017), por la inoperancia de sus sistemas de justicia y por la discrecionalidad permisiva tanto de sus sistemas de gobierno (Meza et al., 2016), como de sus modelos de gobernanza (USAID, 2016); las cuales tienden a mantener su Status Quo (Pelegrini, 2011; Maihold, 2016), como es el caso de Guatemala y Honduras.

Dicha aseveración se sustenta de manera complementaria con los informes de Transparencia Internacional (2017), del Índice de Desarrollo Humano ${ }^{3}$ (2019) y del Banco Mundial (2017). Asimismo, con el surgimiento de la Comisión Internacional Contra la Impunidad en Guatemala (CICIG) y de la Misión de Apoyo Contra la Corrupción y la Impunidad en Honduras (MACCIH), las cuales se crearon en el seno de la Organización de las Naciones Unidas (ONU) y de la Organización de los Estados Americanos (OEA).

Aunque su hegemonía ha sido evidente en contraposición a la corrupción y a la impunidad (Zamudio González, 2018; IUDPAS \& WOLA, 2019), debemos mencionar que ambas han sido objeto de constantes debates y cuestionamientos (Maihold, 2016; FOSDEH, 2019). Es por ello que, esta macro-

3 El Índice de Desarrollo Humano (en adelante: ÍDH) es un instrumento elaborado por el Programa de las Naciones Unidas para el Desarrollo (PNUD), en el que los rangos de evaluación de 2018 se encuentran entre: 0,954 y 0,377, y la aproximación a 1 es favorable (Conceição et al., 2019); contemplando variables como: educación, desigualdad, salud, seguridad y pobreza, entre otras. comparación resulta necesaria para revitalizar su legado, y brindar una mirada alternativa al Status Quo que impera en cada sociedad, ya que las extrapolaciones sobre su eficacia carecen de objetividad.

Sibien se cuenta con estudios especializados como el del Instituto Universitario en Democracia, Paz y Seguridad (2019), el del Instituto Centroamericano de Estudios Fiscales (2015) y el del Foro Social de la Deuda Externa y Desarrollo de Honduras (2019), entre otros. Durante la revisión de literatura, no se encontraron estudios que analicen paralelamente la incidencia de estas entidades, y que, a su vez, determinen su eficacia después del cese de sus mandatos.

Por esta razón, este estudio supone avances innovadores no sólo a nivel democrático y de políticas públicas, sino a nivel científico y jurídico.

En el campo democrático y de políticas públicas, debemos de establecer que al exponer los principales alcances de la CICIG y la MACCIH, se brindan parámetros para fortalecer las democracias de Guatemala y Honduras, ya que permite dilucidar sus barreras estructurales. De manera que, es un aporte doctrinario para juristas, académicos y gestores públicos, y para todos aquellos que conforman la sociedad.

En el campo científico y jurídico, si bien puede pensarse en una simple cotejación subjetiva sobre su legado y normativa, en esta contribución también se exponen los antecedentes de Guatemala y Honduras para contextualizar sus incidencias. De forma que, 
se cumple con el pragmatismo de los estudios jurídicos comparados, al retrotraer sus eventos de manera descriptiva.

\section{METODOLOGÍA}

La metodología es una macro-comparación amparada en las etapas descritas por el ilustre Mauro Cappelletti (Ferrer Mac-Gregor, 2009; González Martín, 2010), la cual permite describir y analizar a la CICIG y a la MACCIH, y asimismo, sus implicaciones dentro de cada realidad social. La pretensión de esta metodología cualitativa es utilizar un marco teórico formal y común para brindar un mayor grado de coherencia interpretativa; y evitar extrapolaciones durante la generación del conocimiento científico.

Las etapas que ofrece el comparatista Mauro Cappelletti (2009), se describen de la manera siguiente:

- La identificación de un punto de partida (tertium comparationis), para determinar la necesidad o problema compartido.

- Determinar normas, instituciones y procesos jurídicos con los cuales los países han intentado resolver el problema, mediante soluciones jurídicas.

- Encontrar las razones que puedan explicar las analogías y diferencias en las soluciones adoptadas en respuesta a un mismo problema.

- La investigación sobre las tendencias evolutivas, las cuales según Eduardo Ferrer Mac-Gregor (2009) puede ser tanto convergentes, como divergentes.
- La valoración sobre las soluciones adoptadas o los modelos de solución en lo que respecta a la eficacia o ineficacia en la resolución del problema.

- En la última fase Cappelletti hace énfasis en la predicción de desarrollos futuros. Sin embargo, ante el cese de la CICIG y la MACCIH se brinda una reflexión desde la ontología jurídica (Sanchéz De la Torre, 1969), desde la axiología jurídica (Chávez Fernandez Postigo, 2017) y desde la filosofía jurídica existencial (Meraz, 2005).

\section{LA IDENTIFICACIÓN DE UN PUNTO DE PARTIDA}

El punto de partida de esta macrocomparación deriva de los acontecimientos de corrupción e impunidad que propiciaron la creación de la CICIG en 2006 (Zamudio González, 2018) y la MACCIH en 2016 (Meza et al., 2016). Aunque su funcionamiento se ha extinguido, como se ha mencionado ambas han sido objeto de constante debate, tanto en el foro nacional, como internacional (Zamudio González, 2018; FOSDEH, 2019), principalmente por las problemáticas que dieron hincapié a su conformación, ya que la corrupción y la impunidad parecen haberse enraizado en el aparato estatal (IUDPAS \& WOLA, 2019).

Asimismo, han sido objeto de cuestionamientos por el tema de la soberanía y la injerencia extranjera (CICIG, 2009; FOSDEH, 2019). Sin embargo, un evento que además de contextualizar ambos escenarios, contrasta dichos cuestionamientos, es el 
hecho que durante la década de los 80 s en estas naciones se promovieron los Programas de Modernización o denominados: Programas de Ajuste Estructural (PAE), impulsados por el Fondo Monetario Internacional y el Banco Mundial (Bonal et al., 2006; Morales Ulloa, 2013).

A pesar que estos programas se promovieron con tintes progresistas y renovadores en la región latinoamericana, se caracterizaron por su descontextualización ante la realidad de las naciones (Bonal et al., 2006). Pero éstas en un periodo de crisis y en su estado más ortodoxo, promovieron su instauración, sin realizar ningún tipo de adecuación contextual (Morales Ulloa, 2013).

Esto muestra ilustrativamente que en estos ordenamientos jurídicos se han implementado políticas compartidas con organismos supranacionales. Aunque en este caso la abstracción fue neoliberal como programas de pseudo modernización, son eventos que cierran el debate sobre la legitimidad de la CICIG y la MACCIH; y más cuando la visión proselitista de los gobiernos en materia de democracia, gobernanza e impartición de justicia mantiene el mismo matiz reduccionista.

El cual ha derivado en impunidad, corrupción y sub-desarrollo. Esto se sustenta de manera complementaria con el informe del ÍDH (2019), en donde Guatemala aparece con 0,651 y Honduras con 0,623 , y con el informe de Transparencia Internacional (2017), en el que Guatemala aparece en el ranking 143 de 180 países y Honduras en el 137. Asimismo, con el indicador de corrupción del Instituto del Banco Mundial (2018), en que Guatemala aparece con la calificación de 22.12 de 100 y Honduras con 29.33.

Por este motivo, la macro-comparación es una necesidad compartida, la cual ha pretendido ofrecer una mirada alternativa al Status Quo, puesto que el surgimiento de la CICIG y la MACCIH no son eventos aislados. Al contrario, develan que la autosuficiencia estatal ha ido en detrimento de su desarrollo; $\mathrm{y}$ en retrospectiva que su asistencia ha sido favorable, dado que, durante su mandato las normas se aplicaron con mayor rigor, veracidad y firmeza en cada sistema jurisdiccional.

Esta aseveración se evidencia por antecedentes protagonizados por la CICIG y por la MACCIH, entre los que destacan casos emblemáticos como el de la Superintendencia de Administración Tributaria (SAT), en Guatemala, dedicada durante años a la defraudación presupuestaria y al lavado de dinero (ICEFI, 2015), y el caso del Instituto Hondureño de Seguridad Social (Meza et al., 2016), con un desfalco millonario, el que derivó en al menos 40 líneas de investigación.

\section{DETERMINACIÓN DE NORMAS, INSTITUCIONES Y PROCESOS JURÍDICOS}

En lo que respecta a determinar las instituciones, normas y procesos con los cuales se ha pretendido resolver el problema, como se ha mencionado se ha definido a la CICIG y a la MACCIH, considerando que, en las praxis ambas entidades han abordado las problemáticas de corrupción e impunidad con gran hegemonía. Indudablemente estos procesos han sido algo distintos, al igual que su génesis. 
En el caso de la CICIG, surgió por medio de un acuerdo entre el Gobierno de Guatemala y la ONU en la ciudad de Nueva York (CICIG, 2008). Posteriormente, sería acreditada para operar en atención a una consulta realizada a los Magistrados de la Corte de Constitucionalidad de Guatemala (DCA, 2007). Al ser favorable, fue ratificada en el Congreso de la república de este país (Zamudio González, 2018).

En lo que respecta a la MACCIH, siguió un protocolo similar, al ser creada mediante un convenio suscrito entre la OEA y el Gobierno de Honduras (OEA \& Estado de Honduras, 2016). En razón de esto, se infiere que ambas surgieron con carácter supranacional, y en atención a protocolos legitimadores, iniciaron sus operaciones. Evidentemente sus prerrogativas son los aspectos más sensibles, en vista que han determinado su nivel de incidencia en los procesos judiciales.

\section{ANALOGÍAS Y DIFERENCIAS EN LAS SOLUCIONES ADOPTADAS}

En cuanto a las razones que explican las analogías y diferencias para resolver las problemáticas de corrupción e impunidad, nos hemos centrado de manera complementaria en aspectos contextuales de cada país, en sus modelos democráticos y en sus ordenamientos jurídicos, considerando que son fundamentales para comprender las implicaciones de la CICIG y de la MACCIH.

Partiendo de sus aspectos contextuales, ambas naciones se han caracterizado por su constante inestabilidad política, en gran parte a consecuencia de sus Golpes de Estado (Meza et al., 2010). En el caso Guatemala (CIA, 1994), el último evento se dio en 1954, con el derrocamiento de Jacobo Arbenz Guzmán mediante una operación denominada: "PBSUCESS", las secuelas de este evento propiciaron el conflicto armado de 1960, el cual perduró hasta la suscripción de los acuerdos de paz en 1996 (Maihold, 2016).

En el caso de Honduras, aunque no llegaron a desencadenarse conflictos bélicos como en Guatemala, desde la década de los 50s hasta la fecha se han suscitado 4 Golpes de Estado. En 1957 al gobierno de Julio Lozano Díaz (Meza et al., 2010); en 1963 con un régimen encabezado por el General Oswaldo López Arellano (CIA, 1963); en 1978 con el triunvirato liderado por el General Policarpo García Paz (Posas, 1980); y en 2009 con el derrocamiento de Manuel Zelaya Rosales (Meza et al., 2016).

La relevancia de estos eventos, es debido a que la inestabilidad se interrelaciona con la corrupción e impunidad (Aidt, 2010; Pelegrini, 2011). No obstante, concurren otros factores como las estructuras de poder en la sociedad (Meza et al., 2016), es por ello que, al estudiar estos contextos es frecuente encontrar apreciaciones como la del profesor Günther Maihold (2016), en la que establece que la impunidad paso por una transición en Guatemala, de una constante de la guerra civil, a una constante de la post-guerra, impulsada por las "CIACS"

4. Los Cuerpos Ilegales y Aparatos Clandestinos de Seguridad (CIACS), surgieron durante el conflicto de 1960. Aunque su cese se esperaba después de los acuerdos de paz en 1996, con sus operaciones posteriores han sido los responsables de sumergir a los guatemaltecos a niveles de violencia e impunidad sin precedentes (Zamudio González, 2018). 
En el caso de Honduras, además de una estructura de poder conformada por políticos, militares y empresarios (Zelaya et al., 2013), sus políticas en materia de democracia, justicia y seguridad se han visto socavadas por sus ciclos atípicos, los cuales tuvieron que haberse implementado de manera paulatina, con revisiones graduales y modificaciones coherentes. Por ejemplo, en menos de una década se creó una Ley para garantizar la Transparencia y el Acceso a la Información Pública (2006) y en el periodo siguiente una Ley de Secretos (2011).

Asimismo, se pueden mencionar otras medidas contradictorias en su ordenamiento jurídico, las cuales aparte de revelar sus ciclos atípicos, muestran un escenario político fraccionado, ante la visión de los gobiernos que se han ido sucediendo en el poder. No obstante, la pretensión no es profundizar en un debate sobre los cambios de la política gubernamental, como se expone en el trabajo de Gustavo Zelaya, Javier Suazo y otros (2013), sino contextualizar ambos escenarios.

Manteniendo de esta forma, el matiz comparatista de René David, descrito por Marta Morineau (2006) al establecer que: "el papel del derecho comparado es parecido al de la historia; dándole al estudioso del derecho nacional la perspectiva necesaria para tener una visión adecuada de los puntos fundamentales y la evolución de su derecho, y permitiéndole, por otro lado, un planteamiento más exacto de los posibles problemas que se presenten, para lograr una mejor solución a las cuestiones jurídicas que se deban resolver" (p. 22).
Por esta razón, al analizar en su conjunto sus transformaciones democráticas, sus ordenamientos jurídicos y los datos descritos del Índice de Desarrollo Humano (2019), de Transparencia Internacional (2017) y del Banco Mundial (2018), ambos contextos pueden calificarse como escenarios distópicos semejantes, en los cuales se denota que tanto la corrupción, como la impunidad se han arrastrado como el espectro histórico de sus modelos anteriores.

\section{TENDENCIAS EVOLUTIVAS}

En lo que respecta a investigar las grandes tendencias evolutivas, nos hemos enfocado exclusivamente en la CICIG y la MACCIH, puesto que la ONU y la OEA las han reconocido casi como híbridos jurídicos, con características particulares y sin antecedentes tipológicos (MACCIH, 2016; CICIG, 2008).

$\mathrm{Si}$ bien la legislación guatemalteca $\mathrm{y}$ hondureña por su tradición colonial puede clasificarse dentro de la familia romanogermánica. Los antecedentes de la Sociedad de las Naciones y de la Comunidad Económica Europea, denominada en la actualidad: Unión Europea, brindan un contraste demasiado amplio. Además de esta disociación, he de añadir que su mandato se ha extinguido, por lo que se ha optado por profundizar en su naturaleza, en su exposición de motivos y en sus contenidos o parte dispositiva.

En lo que respecta a su naturaleza, como se ha mencionado se han seguido protocolos similares para legitimar su funcionamiento. Incluso, la CICIG parece haber ostentado una categoría constitucional, ya que partiendo de la Carta Magna de Guatemala dicho protocolo 
se dio en consonancia con el procedimiento consultivo que establece su legislación (DCA, 2007); contemplando las atribuciones del Congreso Nacional, del Poder Ejecutivo y de la Corte de Constitucionalidad.

Asimismo, el protocolo de la MACCIH contó con un alto grado de legitimidad y de legalidad, dado que, partiendo de sus considerandos (OEA \& Estado de Honduras, 2016), y de los preceptos del Capítulo III de la Constitución de Honduras, su creación se amparó en principios del derecho internacional, y en el procedimiento de los tratados para convertirse en derecho interno.

Pese a esto, ambas entidades operaron al tenor de su enfoque constitutivo, como una Comisión Internacional Contra la Impunidad en Guatemala y como una Misión de Apoyo Contra la Corrupción y la Impunidad en Honduras, lo cual se refleja en su exposición de motivos o considerandos.

En estos apartados también se expone la convulsión sistemática que representan la corrupción y la impunidad en cada sociedad. No obstante, sus pretensiones se esbozan en diferente manera, en el acuerdo de la CICIG con una referencia acusatoria a los cuerpos ilegales de seguridad o denominados: "CIACS" (ONU \& Estado de Guatemala, 2006), y en el convenio de la MACCIH con una referencia general sobre las problemáticas de la corrupción e impunidad (OEA \& Estado de Honduras, 2016).

Otra distinción se refleja con el auxilio solicitado a la ONU y a la OEA para su creación, en la CICIG, sus considerandos demuestran una asistencia solicitada por el gobierno guatemalteco (ONU \& Estado de Guatemala, 2006). En la MACCIH por los reclamos de la sociedad hondureña, evidentemente el planteamiento utilizado en la MACCIH es un eufemismo, después que el gobierno admitiese que se sustrajeron fondos del IHSS para financiar campañas políticas (CESPAD, 2019).

Incluso, en principio las manifestaciones exigieron una comisión anti-corrupción a la ONU (CESPAD, 2019). No obstante, su establecimiento se realizó con evidentes adecuaciones en relación con la CICIG, reflejadas no sólo con sus protocolos y sus considerandos, sino con su contenido o parte dispositiva, principalmente en los apartados sobre sus objetivos, sobre sus funciones y competencias y sobre el papel o rol de la nación contraparte.

En lo que respecta a sus objetivos, se formularon en apego a otros acuerdos emblemáticos para fortalecer sus democracias, como el Acuerdo Global sobre Derechos Humanos para Guatemala (ONU \& Estado de Guatemala, 2006) y la Convención de las Naciones Unidas Contra la Corrupción para Honduras (OEA \& Estado de Honduras, 2016).

En cuanto al acápite sobre sus funciones, en ambos casos se alinearon con sus pretensiones, en la CICIG, con recomendaciones al gobierno para adoptar políticas para erradicar y desmantelar estructuras criminales. En la MACCIH, sus funciones son más amplias, con una taxonomía sobre la prevención y el combate a la corrupción, sobre la justicia 
penal, sobre la política electoral y sobre la seguridad pública.

Sin embargo, a nivel de facultades y de competencias, la CICIG además de contar con un perfil como ente asesor, aparece en calidad de: "Querellante Adhesivo", acorde con las facultades establecidas en la legislación guatemalteca. Lo cual posiblemente es la disparidad principal, en vista que el accionar de la MACCIH fue limitada en ese sentido, puesto que su marco de competencia fue estrictamente dictaminador.

En lo referente al apartado sobre el rol o papel de los Estados, en términos generales fue similar al establecer garantías esenciales para el cumplimiento de la CICIG y la MACCIH, al tenor de lo estipulado en sus respectivos estatutos. Esto se materializo en Guatemala con la creación del Observatorio Judicial del Sistema de Justicia Penal y del Sistema Integrado de Justicia (CICIG, 2019), y en Honduras con el Observatorio de Justicia Penal y con el Plan de Comunicación Estratégica (MACCIH, 2019).

Incluso, el acuerdo de la CICIG hace referencia a la coordinación con el gobierno para hacer efectivo el rol de: "Querellante Adhesivo", lo cual data en más de 100 casos (CICIG, 2018). Sin embargo, una divergencia filológica a mencionar es la connotación utilizada en estos apartados, aparecen como: "Art. 6. Cooperación con la CICIG" y "Art. VIII. Obligaciones del Gobierno de Honduras", ya que la cooperación se refleja como una colaboración incondicional del Estado, y la obligación como un término propenso a incumplimiento.

\section{VALORACIÓN SOBRE LAS SOLUCIONES ADOPTADAS}

En esta fase Mauro Cappelliti establece que deben evaluarse las soluciones adoptadas para determinar la eficacia o ineficacia en la resolución del problema, pero considerando que la corrupción y la impunidad son una consecuencia histórica y contextual de cada Estado. En este apartado se han contemplado los resultados obtenidos en los informes oficiales de la CICIG y la MACCIH, los cuales en su conjunto permiten calificar su incidencia de manera efectiva.

Entre los logros de la CICIG que permiten emitir dicha valoración, destacan sus acciones para sensibilizar a la sociedad guatemalteca sobre la problemática de corrupción e impunidad, y el trabajo con la Policía Nacional Cívica para devolver la confianza a la ciudadanía (CICIG, 2009, 2013). Asimismo, las auditorias sociales en las postulaciones de los Magistrados de la Corte Suprema de Justicia, de la Corte de Apelaciones, del Director del Instituto de la Defensa Pública Penal y del Fiscal General (CICIG, 2011).

Además de esto, las constantes recomendaciones para realizar reformas legislativas (CICIG, 2012,2017), la estabilidad brindada al Ministerio de Gobernación (CICIG, 2011), la transferencia de capacidades al Ministerio Público, las 1,200 personas condenadas por actos de corrupción (CICIG, 2017, 2019), y posiblemente el evento más emblemático, el desmantelamiento de la estructura criminal que llevo al General Otto Pérez a la Presidencia de la República y a Roxana Baldetti Elías a la Vicepresidencia (CICIG, 2015). 
En lo que respecta a la MACCIH, destaca la creación de Jueces con Jurisdicción Nacional en materia Anti-corrupción (MACCIH, 2016), la creación de una ley electoral, conocida como: "Ley de política Limpia" para prevenir las irregularidades de los partidos políticos (MACCIH, 2016), y una Ley de Colaboración Eficaz, con regulaciones similares a las utilizadas en Perú para desarticular la red de "Fujimori y Montesinos", en Brasil para el caso "Odebrecht" y en Guatemala para desarticular a las "CIACS" (MACCIH, 2017).

Entre otros logros se puede hacer referencia de colaboraciones a la Unidad Fiscal Especial Contra la Impunidad de la Corrupción (UFECIC), como en el caso del IHSS, en donde se obtuvo la condena más rigurosa a un alto funcionario por actos de corrupción (MACCIH, 2017). Asimismo, colaboraciones en casos emblemáticos como: la "Red de Diputados", la "Caja Chica de la Dama", el "Caso Pandora" y el "Proyecto de Agua Zarca", este último es concerniente al asesinato de la ambientalista Berta Cáceres (MACCIH, 2016).

En definitivo, ante tal hegemonía estas entidades han recibido el apoyo de ambas sociedades, y de manera análoga, el apoyo de la comunidad internacional. No obstante, las complejidades contextuales de cada nación han sido los aspectos que han obstaculizado su accionar, y por consiguiente limitado su eficacia.

Por ejemplo, la CICIG a pesar de realizar constantes recomendaciones a nivel de políticas públicas, según su informe de 2012, hasta ese periodo sólo se realizaron 6 de 20 reformas (CICIG, 2012). De manera que, la voluntad política de los parlamentarios ha sido un impedimento ostensible, debiendo agregar que las reformas se señalaron en reiteradas ocasiones como aspectos fundamentales para fortalecer el Estado de derecho y asegurar las garantías democráticas en la impartición de justicia (CICIG, 2016, 2017, 2018).

Otro aspecto en el caso guatemalteco, y posiblemente el más complejo es la evolución de la "CIACS" como Red Político-Económica Ilícita (RPEI) (Zamudio González, 2018). Según informes de la CICIG (2016, 2017, 2018), dicha mutación criminal utiliza nuevos mecanismos, como contra-inteligencia para consolidar sus prácticas delictivas, y consecuentemente su impunidad; esto con la ayuda de políticos como gestores de influencias (CICIG, 2015), los cuales se encuentran dentro de las estructuras de poder.

En el caso de la MACCIH, su mandato se ha visto socavado por su corta vigencia, y por el periodo de inestabilidad a consecuencia de la crisis post-electoral en 2017 (MACCIH, 2018a), la cual aparte de afectar su operatividad, retraso la discusión de varias reformas. Asimismo, hay que añadir los constantes cambios a nivel directivo, primero con el Abog. Juan Jiménez Mayor (MACCIH, 2017), luego con la Fiscal Ana María Calderón Boy (MACCIH, 2018a) y para finalizar con el Dr. Luiz Guimaraes Marrey (MACCIH, 2018b).

Aunque estos cuentan con trayectorias reconocidas nivel nacional e internacional en favor de la democracia y de la justicia, sus transiciones nivel directivo no pueden calificarse de manera favorable en un periodo 
tan fugaz. Además, si contemplamos que después del cese de su mandato, la UFECIC fue desarticulada de manera radical, y remplazada por la UFERCO (La Prensa, 2020a, 2020b).

\section{PREDICCIÓN DE DESARROLLOS FUTUROS / REFLEXIÓN}

En su conjunto el contraste de estos acontecimientos es lo que nos ha llevado a la reflexión sobre la CICIG y la MACCIH, ya que, pese a su hegemonía, es indudable la necesidad de revitalizar su legado. Por esta razón, ambas entidades han sido analizadas desde la perspectiva ontológica de Ángel Sánchez de la Torre (1969), desde la perspectiva axiológica de Luis Recaséns Siches (Chávez Fernandez Postigo, 2017) y desde la perspectiva de la filosófica de Esteban Krotz (Meraz, 2005).

Partiendo de la ontología jurídica, debemos mencionar que antes de su génesis ambas entidades fueron concebidas de manera abstracta, pero con su entrada en vigor se convirtieron en parte de cada contexto, considerando que incidieron en las problemáticas de corrupción e impunidad. Aunque es evidente que tanto sus enfoques, como competencias son distintos, su preminencia progresista es innegable, y esto les ha permitido sobrepasar el imaginario subjetivo de la norma, posicionándolas dentro de cada realidad social.

Sin embargo, para Ángel Sánchez de la Torre (1969), estos posicionamientos se crean no sólo por su incidencia en dichas problemáticas, sino por aspectos como la opinión pública de la ciudadanía, las posturas de sus detractores políticos y la dialéctica mantenida con los gobiernos; materializadas en colaboraciones y en acciones jurisdiccionales. Otro aspecto a destacar son sus interconexiones con el bien común, el cual debe primar en toda sociedad, y que se refleja plenamente con su enfoque y su modo operandi.

En lo que respecta a la Axiología Jurídica, debemos establecer que los protocolos para su creación muestran consonancia con los ordenamientos jurídicos. Si bien sus detractores con aparentes posturas nacionalistas emitieron señalamientos sobre la violación de la soberanía (FOSDEH, 2019), parecen no más que resistencias por parte de las estructuras de poder. En vista que la armonía normativa se evidencia con sus prelaciones estatutarias, las cuales han marcado la pauta de su dinámica jurídica.

Por ejemplo, la CICIG pese a contar con facultades como: "Querellante Adhesivo", mantuvo sus acciones dentro de su enfoque constitutivo, dado que no incoó acciones afuera del marco de su competencia, lo cual consta en sus informes oficiales. Atributo compartido con la MACCIH, ya que en el caso de ésta no participo como parte acusadora, lo que devela que el principio Pacta Sunt Servanda ha sido parte de sus fundamentos. Esto también se sustenta al no renovar sus mandatos sin el consentimiento de sus contrapartes.

En cuanto a la filosofía jurídica existencial (Meraz, 2005), ambas comparten matices existencialistas, pero con marcadas peculiaridades. Por ejemplo, en su génesis la CICIG surgió en respuesta a una petición 
gubernamental (Zamudio González, 2018), y su mandato se vio fortalecido con sus hallazgos, como con el caso de: "Cooptación del Estado", el cual derivó en la condena de Roxana Baldetti Elías y en la dimisión del General Otto Pérez a la Presidencia de República (CICIG, 2015).

Estoshallazgos propiciaron manifestaciones durante 4 meses en la sociedad guatemalteca (CICIG, 2015). En cambio, la MACCIH, como se ha mencionado surgió por manifestaciones de la sociedad (MACCIH, 2016). Por esta razón, se devela que su divergencia existencial radica en la anuencia gubernamental; también reflejada con la renovación de la CICIG en 4 ocasiones (CICIG, 2017), mientras que el mandato de la MACCIH no se renovó, pese a contar con una coalición para este cometido (FOSDEH, 2019).

\section{RESULTADOS}

1. Sintetizado los hallazgos de manera cronológica, se puede establecer que la corrupción y la impunidad se han acentuado como una simbiosis sistemática. Los datos descritos de Transparencia Internacional (2017), del índice de Desarrollo Humano (2019) y del Banco Mundial (2018) sustentan esta premisa. Es por ello que, esta macro-comparación resulta tanto una necesidad compartida, como diferenciada, en vista que la CICIG y la MACCIH contaron con mecanismos para abordar dichas problemáticas.

2. Asimismo, los datos de estas organizaciones, y los aspectos contextuales de ambas naciones han permitido definir a la CICIG y a la MACCIH como las instituciones objeto de análisis, puesto que con su hegemonía protagonizaron avances notables en cada ordenamiento jurídico; y más allá de su consonancia articuladora con cada legislación, su legitimidad se ha visto reflejado con el respaldo de la sociedad guatemalteca y de la sociedad hondureña.

3. En lo que respecta al tema de las coincidencias y divergencias sobre las soluciones adoptadas, es imprescindible traer a colación la inestabilidad histórica caracterizadas por estas naciones. A pesar de que sus antecedentes son distintos, son aspectos que han determinado la evolución de los ordenamientos jurídicos, y consecuentemente la fragilidad de los sistemas democráticos; lo cual ha pretendido fortalecerse con las diversos competencias de la CICIG y la MACCIH.

4. En lo concerniente al análisis sobre su naturaleza, exposición de motivos y contenidos, debemos establecer que surgieron en respuesta a las mismas problemáticas. Pero con diferencias patentizadas en formas más allá de sus protocolos de instauración, dicha aseveración se aprecia principalmente con el enfoque acusatorio en los considerandos de la CICIG, y con sus facultades como: "Querellante Adhesivo", ya que la MACCIH no contó con dicha competencia $\mathrm{y}$ tampoco con un enfoque personalizado.

5. Pese a estas distinciones, al valorar sus operaciones en el marco de su competencia, ambas entidades pueden calificarse 
como efectivas, dado que sus avances han sido un contrapeso para erradicar las problemáticas. Además de esto, su transferencia de capacidades ha sido un aliciente para fortalecer los sistemas de justicia. No obstante, es innegable el escepticismo ante el incumplimiento de las reformas recomendadas, y ante los cambios subsecuentes generados en sus sistemas.

6. En cuanto a la reflexión, contemplando los hallazgos en este estudio se han revelado tres aspectos relevantes, a nivel ontológico, que su interacción las ha posicionado dentro de cada realidad social; a nivel axiológico, que existe una consonancia normativa preponderante con los Estados, puesto que su instauración $\mathrm{y}$ funcionamiento se amparó al tenor de cada legislación. Sin embargo, a nivel existencialista, pese a compartir ciertos matices, se detecta una divergencia sensitiva, y radica en la voluntad política para ampliar sus mandatos, evidentemente esto depende de agentes externos.

\section{DISCUSIÓN}

En lo que respecta a la discusión de resultados, debemos establecer que en este estudio se han revelado aspectos que han permitido entender la naturaleza, el enfoque y el funcionamiento de la CICIG y la MACCIH.

Sin embargo, en atención a los resultados, es oportuno pensar si es posible mejorar los sistemas democráticos, cuando históricamente en las más altas esferas se han encontrado los artífices de la corrupción y la impunidad.
Esto se trae a colación considerando que los esfuerzos de la CICIG y la MACCIH podrían disiparse, tal como sucedió en el caso hondureño con la UFECIC. Incluso, esto abre un nuevo debate sobre los límites de la soberanía en contextos caracterizados por su inestabilidad política.

Otro aspecto significativo a contemplar, es cómo manejar la credibilidad de las instituciones que han recibido la transferencia de capacidades. Aunque sus mecanismos pueden ser igual de determinantes que los de la CICIG y la MACCIH, el rechazo de la sociedad puede minimizar sus actuaciones. Por esta razón, los nuevos criterios de efectividad deben ser muy objetivos, y enfatizar en su autonomía, considerando que la opinión pública clama con fervor por la incoación de acciones con total independencia.

Además de esto, es cuando deben contemplarse las bondades de la CICIG y la MACCIH como entidades pioneras, ya que, pese a sus divergencias, tanto sus enfoques, como competencias han abordado la prevención, regulación y erradicación de las problemáticas de la corrupción y la impunidad. Las anomias que a todas las luces siguen socavado los modelos estatales. Debiendo añadir que, su incidencia también brindo la armonía normativa que debe prevalecer en toda sociedad.

No obstante, como limitaciones en este estudio se debe enunciar la vigencia de las entidades, puesto que la CICIG opero de 2007 a 2019 y la MACCIH de 2016 a 2020. Por esta razón, la comparación sobre su nivel impacto 
ha sido restringido, dado que el periodo de vigencia coincidente ha sido demasiado corto. Debiendo sumar a esto, la afectación de la crisis post-electoral en Honduras y la dimisión presidencial en Guatemala, en vista que son eventos que requieren otro tipo de análisis.

Con el tema de las implicaciones que supone para investigaciones futuras, debemos establecer que este proyecto se suma a la generación del conocimiento científico sobre estudios jurídicos comparados. Asimismo, abre nuevas líneas de investigación para profundizar en la CICIG y la MACCIH, y en temas concernientes sobre sus protocolos de instauración, sobre sus marcos normativos, sobre sus competencias y sobre su legalidad y legitimidad.

\section{CONCLUSIONES}

1. En lo que respecta a esta macrocomparación, es razonable concluir que este proyecto ha permitido contextualizar el papel de la CICIG y la MACCIH, revelado su incidencia dentro de cada sociedad, y dejando entrever que su efectividad no ha sido un simple vaticinio. Estas valoraciones se amparan en la materialización de sus enfoques y de sus competencias, plasmadas en colaboraciones y en recomendaciones, $\mathrm{y}$ en la erradicación de diferentes estructuras criminales.

2. Por otra parte, debemos mencionar que la literatura ha revelado que los ordenamientos jurídicos evolucionan al igual que las problemáticas de corrupción e impunidad. Por este motivo, el estudio sobre su legado debe ser una constante para los agentes de cambio que conforman la sociedad, ya que ambas entidades brindaron asistencia para erradicar estas problemáticas. Debiendo agregar, que también han develado el amilanamiento de las estructuras criminales, con las posturas de sus detractores políticos.

3. Si bien los datos descritos del Índice de Desarrollo Humano, de Transparencia Internacional y del Banco Mundial muestran escenarios distópicos, el contrapeso protagonizado por estas entidades también ha revelado una mirada alternativa al Status Quo. Aunque sus divergencias se han reflejado en este proceso, su aceptación, legitimidad e incidencia han sido los fundamentos clave para determinar su eficacia, considerando que han servido como conductores democráticos para fortalecer el Estado de Derecho.

4. Para finalizar debemos establecer que este proyecto comparatista ha develado las carencias y contrariedades que supone el Status Quo y el hermetismo estatal. Es por ello que, como recomendación se exhorta además de profundizar en el legado de la CICIG y de la MACCIH, a cuestionar en la inflexión de los gobiernos sobre las nuevas innovaciones jurídicas, y más aún cuando la asistencia de estas entidades ha incidido favorablemente de en ambos sistemas. 


\section{BIBLIOGRAFÍA}

Aidt, T. S. (2010). "Corruption and Sustainable Development". International Handbook on the Economics of Corruption, Vol. 2, (1-52). Cheltenham, Edward Elgar Publishing.

Bonal, X., Adelantado, J., Arnove, R., Bogliaccini, J., Bracho, T., Calero, J., Escardíbul, J., Filgueira, F., GarciaHuidobro, J., Lazzarotti, B., Lijtenstein, S., López, N., Medieta, G., Raczynski, D., Rambla, X., Rivero, J., Rodríguez, F., Tarabini-Castellani, A., Tedesco, J., Valiente, Ó. \& Verger A. (2006) Globalización, Educación y Pobreza en América Latina ¿Hacia una nueva agenda política?, Barcelona, Bellaterra, S.L.

CESPAD (2019). Cronología/ Resurgen las antorchas en Honduras para exigir la salida de Juan Hernández. Centro de Estudio Para La Democracia. Recuperado de https:// cespad.org.hn/2019/04/08/cronologiaresurgen-las-antorchas-en-honduras-paraexigir-la-salida-de-juan-hernandez

Chávez Fernandez Postigo, J. C. (2017) La dimensión axiológica de la filosofía de la interpretación del derecho de Luis Recaséns Siches: una revaloración a la luz de la teoría estandar de la argumentación jurídica, [Universidad de Zaragoza].

CIA (1963). Telegram information reportCentral Intelligence Agency https:// www.archives.gov/files/declassification/ iscap/pdf/2012-168-doc3.pdf
CIA (1994). Operation PBSUCCESS. Central Intelligence Agency https:// www.cia.gov/library/readingroom/docs/ DOC 0000134974.pdf

CICIG (2008). I Informe Anual de Labores de la Comisión Internacional contra la Impunidad en Guatemala. Ciudad de Guatemala, Edición CICIG.

CICIG (2009). II Informe Anual de Labores de la Comisión Internacional contra la Impunidad en Guatemala. Ciudad de Guatemala, Edición CICIG.

CICIG (2011). IV Informe Anual de Labores de la Comisión Internacional contra la Impunidad en Guatemala. Ciudad de Guatemala, Edición CICIG.

CICIG (2012). V Informe Anual de Labores de la Comisión Internacional contra la Impunidad en Guatemala. Ciudad de Guatemala, Edición CICIG.

CICIG (2015). VIII Informe Anual de Labores de la Comisión Internacional contra la Impunidad en Guatemala. Ciudad de Guatemala, Edición CICIG.

CICIG (2016). IX Informe Anual de Labores de la Comisión Internacional contra la Impunidad en Guatemala. Ciudad de Guatemala, Edición CICIG.

CICIG (2017). X Informe Anual de Labores de la Comisión Internacional contra la Impunidad en Guatemala. Ciudad de Guatemala, Edición CICIG. 
CICIG (2018). XI Informe Anual de Labores de la Comisión Internacional contra la Impunidad en Guatemala. Ciudad de Guatemala, Edición CICIG.

CICIG (2019). XII Informe Anual de Labores de la Comisión Internacional contra la Impunidad en Guatemala. Ciudad de Guatemala, Edición CICIG.

Conceição, P., Assa, J., Calderón, C., Gray, R., Gulasan, N., Hsu, Y.-C., Kovacevic, M., Lengfelder, C., Lutz, B., Mukhopadhyay, T., Nayyar, S., Palanivel, T., Rivera, C., \& Tapia, H. (2019). Informe sobre Desarrollo Humano 2019, Nueva York, NY10017, Programa de las Naciones Unidas para el Desarrollo.

Congreso Nacional de República de Honduras (2011). Ley de Secretos Oficiales y de Clasificación de la Información, Tegucigalpa.

Congreso Nacional de República de Honduras (2006). Ley de Transparencia y Acceso a la Información Pública, Tegucigalpa.

DCA, Diario de Centro América (2007, 10 de septiembre). Consulta favorable de la Corte de Constitucionalidad. Diario de Centro América, (1). https://www.cicig.org/ wp-content/uploads/2018/03/28082007 Ratificacion CICIG.pdf

Ferrer Mac-Gregor, E. (2009). Mauro Cappelletti y el Derecho procesal constitucional comparado. 1 Congreso Mexicano de Derecho Procesal
Constitucional, (325-342). Monterrey, Instituto de Investigaciones Jurídicas de la UNAM y el Instituto Mexicano de Derecho Procesal Constitucional.

FOSDEH (2019). Porqué nació la MACCIH $\&$ porqué debe continuar. Foro Social de Deuda Externa y Desarrollo de Honduras. Tegucigalpa. Recuperado de https://fosdeh. com/wp-content/uploads/2019/10/porquenacio-la-maccih.pdf

González Martín, N. (2010). Sistemas jurídicos contemporáneos. Ciudad de México. In Cultura Jurídica, Nostra Ediciones S. A. de C. V.

ICEFI (2015). Diagnóstico y propuesta alternativa de hoja de ruta para el rescate y reconstrucción de la SAT. Instituto Centroamericano de Estudios Fiscales, Ciudad de Guatemala. Recuperado de https://icefi.org/sites/default/files/ diagnostico y propuesta de hoja de ruta para el rescate y reconstruccion de la sat gt 05-2015.pdf

IUDPAS (2019). La lucha contra la impunidad. Instituto Universitario en Democracia, Paz y Seguridad. Recuperado de https://www. wola.org/wp-content/uploads/2019/10/ Impunidad-HN-ESP-10.9.pdf

La Prensa (2020a, 21 de enero). Funciones de UFECIC terminaron al renovarse el convenio de la MACCIH. La Prensa. Recuperado de https://www.laprensa. hn/honduras/1350662-410/funcionesufecic-terminaron-no-renovarse-convenio- 


\section{maccih-corrupcion-honduras}

La Prensa (2020b, 24 de enero). UFERCO sustituira a la UFECIC para investigar la corrupción en Honduras. La Prensa. Recuperado de https://www.laprensa.hn/ honduras/1351405-410/uferco-sustituiraufecic-investigacion-corrupcion-honduras

MACCIH (2016). Primer Informe Semestral de la Misión de Apoyo contra la Corrupción y la Impunidad en Honduras. Tegucigalpa, Edición MACCIH.

MACCIH (2017). Tercer Informe Semestral de la Misión de Apoyo contra la Corrupción y la Impunidad en Honduras. Tegucigalpa, Edición MACCIH.

MACCIH (2018a). Cuarto Informe Semestral de la Misión de Apoyo contra la Corrupción y la Impunidad en Honduras. Tegucigalpa, Edición MACCIH.

MACCIH (2018b). Quinto Informe Semestral de la Misión de Apoyo contra la Corrupción y la Impunidad en Honduras. Tegucigalpa, Edición MACCIH.

MACCIH (2019). Sexto Informe Semestral de la Misión de Apoyo contra la Corrupción y la Impunidad en Honduras. Tegucigalpa, Edición MACCIH.

Maihold, G. (2016). Intervention by invitation? Shared sovereignty in the fight against impunity in Guatemala. European Review of Latin American and Caribbean Studies, Vol. 101, (5-31).
Meraz, A. (2005). Sobre Antropología Jurídica: Perspectivas Socioculturales en el estudio del Derecho de Esteban Krotz. Isonomía. Revista de Teoría y Filosofía Del Derecho, Vol. 23, (185-190).

Meza, V., Meza, M., Romero, R., Gamero, M., Orellana, E., Llopis, J., García, F., \& Salomón, L. (2016). El manejo político y social de la corrupción en Honduras. Tegucigalpa, CEDOH.

Meza, V., Romero, R., Funes, L., Gamero, M., Salomón, L., \& Murga, A. (2010). Golpe de Estado: Partidos, instituciones y cultura política. Tegucigalpa, CEDOH.

Morales Ulloa, R. (2013). Los cambios en la gobernanza del sistema educativo en Honduras: la política de desconcentración de la educación pre-básica, básica y media (1990-2010), [Universidade do Porto].

Morineau, M. (2006). Evolución de la Familia Jurídica Romano-Canónica, El Derecho Comparado. Sistemas Jurídicos y Derecho Comparado. 2da Edición, (17-45) Ciudad de México, Instituto de Investigaciones Jurídicas de la UNAM.

OEA \& Estado de Honduras (2016). Convenio entre la república de Honduras y la Secretaria General de la Organización de los Estados Americanos para el Establecimiento de la Misión de Apoyo contra la Corrupción y la Impunidad en Honduras (MACCIH). Recuperado de https://www.oas.org/documents/spa/press/ convenio-MACCIH-1.19.16.pdf 
ONU \& Estado de Guatemala (2006). Acuerdo entre la Organización de las Naciones Unidas y el Gobierno de la república de Guatemala relativo al establecimiento de una Comisión Internacional Contra la Impunidad en Guatemala (CICIG). Recuperado de https://www.cicig.org/ uploads/documents/mandato/cicig acuerdo.pdf

Pelegrini, L. (2011). Corruption, Development and Environment. London. Springer Science+Business Media B.V. 201.

Posas, M. (1980). “Tendencias ideológicas actuales en el movimiento obrero hondureño", (25-54). Tegucigalpa, Anuario de Estudios Centroamericanos.

Sanchéz De la Torre, A. (1969).'La Ontologia Juridica Como Filosofia", (83-105). Ciudad de México, Anuario de Filosofía Del Derecho.

Trasparencia Internacional (2017). Corruption Perceptions Index. Berlin, In Encyclopedia of Governance.

USAID (2016). Country Development Cooperation Strategy (2015-2020). Washington D.C. United States Agency for International Development United.

World Bank (2017). World Development Report:Governanceand thelaw. Washington D.C. World Bank Publications.

Zamudio González, L. (2018). La Comisión Internacional contra la Impunidad en Guatemala (CICIG). Una organización autodirigida. Revista Trimestral por El Colegio de México, Vol. 58, (493-535).

Zelaya, G., Suazo, J., Suazo Rubí, S., \& Borjas, J. A. (2013). Elecciones en Honduras: ¿Continuidad, cambio o ruptura después del golpe de Estado ?, Tegucigalpa, Friedrich-Ebert-Stiftung FES. 